\title{
Eine einfache, neue kolorimetrische Pregnandiol-Bestimmungsmethode
}

\author{
Von St. Ilca, C. Dodica und Z. Ionnovici \\ Aus der Rumänischen Akademie der Wissenschaften und der geburtshilflich-gynäkologischen Klinik, Lugoj, Rumänien
}

(Eingegangen am 16. Juni 1970)

Es wird eine neue, einfache Methode zur Bestimmung von Pregnandiol beschrieben, die sich bei der Uberwachung gefährdeter Schwangerschaften berwährt hat. Nach Säurehydrolyse wird mit Toluol extrahiert und aus dem Extrakt nach Verdampfen des Toluols Pregnandiol in äthanol. $\mathrm{NaOH}$ abgeschieden. Der in einer Farbreaktion mit Vanillin gebildete Farbstoff wird bci 510 bis $550 \mathrm{~nm}$ gemessen. Empfindlichkeit, Zuverlässigkeit in der Serie und Wiederfindung sowie Normalwerte für einzelne Schwangerschaftsmonate werden mitgeteilt.

\section{A simple nen colorimetric method for the determination of pregnanediol}

A new, simple method is described for the determination of pregnanediol, which has proved suitable for the surveillance of threatened pregnancies. The urine is acid-hydrolysed and extracted with toluene; the toluene is removed by evaporation and the pregnanediol taken up in ethanolic $\mathrm{NaOH}$. Reaction with vanilline gives a colour which is measured at $510-550 \mathrm{~nm}$. The sensitivity, reliability in serial determinations, and the recovery are reported, together with normal values for each month of pregnancy.

Pregnandiol ist das wichtigste Stoffwechselprodukt von Progesteron und wird in wasserlöslichem Zustand als Glucuronid mit dem Harn ausgeschieden. Heute ist allgemein anerkannt, daß die Pregnandiolausscheidung mit der Progesteronsekretion korreliert ist und daher als wertvoller Index der Funktion des Corpus luteum oder - während der Schwangerschaft — der Plazenta anzusehen ist. Dadurch wird das Interesse für einfache und empfindliche Pregnandiolbestimmungsmethoden verständlich.

Die heute gebräuchlichsten Bestimmungsmethoden weisen im allgemeinen drei typische Schritte auf:

1. die Hydrolyse des Pregnandiol-Glucuronids, welche als konventionelle Säurehydrolyse oder enzymatische Spaltung ausgeführt wird. Der Vorteil der Säurehydrolyse liegt in der Zeitersparnis, ihr Nachteil in der Bildung stark gefärbter Spaltprodukte. Die enzymatische Spaltung wirkt schonender, es bilden sich schwächer gefärbte Spaltprodukte, ihr Nachteil ist die lange Reaktionsdauer.

2. die Reinigung des Extraktes, die eines der Hauptprobleme der Bestimmung ist, denn eine ungenügende Entfernung der Harnchromogene kann sich ungünstig auf das Endresultat auswirken. Dies ist der Grund, weshalb eine große Anzahl Extraktreinigungsverfahren ausgearbeitet wưrden. Dazu zählen das Waschen des Harnextraktes mit Alkali oder alkalischem Permanganat (1), die Adsorptionschromatographie an Aluminiumoxid $(1,2)$ oder an Silicagel (3), die Papierchromatographie $(4,5)$, die Dünnschichtchromatogtaphie (6), die Reinigung durch Aktivkohle (7) oder durch Hexamethylentetramin (8).

3. den Nachweis des Pregnandiols. Zur Bestimmung der Pregnandiolkonzentration benutzt vaN DER MOLEN (9) das Absorptionsspektrum im Infrarot, HENRY und Mitarbeiter (10) im Ultraviolett. Als einfachste Methode ist allerdings die Farbreaktion mit Schwefelsäure anzusehen (11), gegebenenfalls unter Zusatz von Natriumbisulfit (4) oder Glykolsulfit (12). Die Physikochemie der Schwefelsäurereaktion ist nach SAS (13) noch nicht aufgeklärt. Stoffe mit einer oxydativen Potenz, eventuell selbst die Lösungsmittel, können die Farbintensität beeinflussen (13).

Es ist somit klar, daß eine vorbildliche Pregnandiolbestimmungsmethode folgende Eigenheiten erfüllen $\mathrm{muß:}$

eine kurze Hydrolysedauer

eine einfache Reinigung des Extraktes und eine möglichst spezifische Farbreaktion.

Die von uns hier vorgeschlagene Pregnandiolbestimmungsmethode soll diesen Erfordernissen größtenteils nachkommen.

\section{Prinzip}

Nach einer kurzen Säurehydrolyse wird der Toluolextrakt mit Alkali gewaschen, das Toluol vertrieben, aus dem Rückstand das Pregnandiol ausgeschieden. Mit dem ausgeschiedenen Pregnandiol wird die Sulfophospho-Vanillin-Reaktion (14) ausgeführt. Da das verwendete Reagenz die Farbreaktion nur mit Pregnandiol ergibt, werden die noch mitlaufenden Verunreinigungen von der Messung ausgeschlossen. Diese Technik eignet sich besonders zur Überwachung labiler, über 6 Wochen alter Schwangerschaften.

\section{Reagenzien}

1. Salzsäure konz. p. a.

2. Toluol reinst

3. Natriumhydroxid-Lösung, $1 \mathrm{~N}$

4. Athylalkohol reinst

5. Natriumhydroxid-Lösung, $0,1 \mathrm{~N}$

6. Schwefelsäure konz. p. a. $D=1,84$

7. Phospho-Vanillin-Reagenz: $0,12 \mathrm{~g}$ Vanillin werden in $100 \mathrm{ml}$ Orthophosphorsäure $(83-85 \%)$ gelöst. In dunkler Flasche bei Raumtemperatur aufbewahren. 


\section{Ausführung}

In eincm Erlenmeyerkolben werden $200 \mathrm{ml}$ 24-Stdn.-Harn, $35 \mathrm{~m} /$ Toluol und einige Siedsteinchen unter Rückflußkühlung zum Sicden erhitzt. Dann werden - am zweckmäßigsten durch den Kühler - sehr langsam und vorsichtig $20 \mathrm{~m} / \mathrm{konz}$. Șalzsäure zugesetzt. Man läßt genau $10 \mathrm{Min}$. sieden, kühlt sofort ab, entfernt im Scheidetrichter die wäßr. Phase und verwirft sie. Zur Abstumpfung der im Extrakt verbliebenen Säure und zur Entfernung sämtlicher alkalilöslicher Produkte wäscht man $2 \mathrm{mal} \mathrm{mit}$ je $15 \mathrm{~m} / 1 \mathrm{~N}$ Natronlauge, zuletzt mit $15 \mathrm{ml}$ dest. Wasser. Die wäßr. Phase wird jedesmal verworfen. Der Toluolextrakt wird durch ein mit Toluol angefeuchtetes Filterpapier (wobei er gleichzeitig entwässert wird) filtriert, danach wird das Toluol im siedenden Wasserbad vertrieben. Der trockene Rückstand wird in $5 \mathrm{~m} l$ Äthylalkohol gelöst. Zu $1 \mathrm{~m} l$ dieses alkoholischen Extraktes gibt $\operatorname{man} 5 \mathrm{ml} 0,1 \mathrm{~N}$ Natronlauge und scheidet das Pregnandiol durch kurzes Erhitzen ( 90 Sek.) im siedenden Wasserbad aus. Man läßt abkühlen, zentrifugiert nach 30 Min. kräftig, gießt $a b$, läßt abtropfen, gibt zum Rückstand $4 \mathrm{~m} l$ konz. Schwefelsäure, schüttelt bis zur Lösung und erhitzt im siedenden Wasserbad genau 10 Min., kühlt rasch ab.

In ein Proberöhrchen gibt man $2 \mathrm{~m} /$ Phospho-Vanillin-Reagenz, dazu genau $0,1 \mathrm{ml}$ des Schwefelsäure-Pregnandiol-Reaktionsgemisches, rührt gut um und läßt $20 \mathrm{Min}$. bis zur Entwicklung der rosa Farbe stehen. Nachher wird kolorimetriert. Schichtdicke $0,5 \mathrm{~cm}$, Filter $510-550 \mathrm{~nm}$, Extinktionsmaximum $=530 \mathrm{~nm}$. Die Extinktion der Analysen wird gegen das Phospho-VanillinReagenz gemessen, die Eichkurve mit einer geeigneten Standardlösung von Pregnandiol aufgestellt.

\section{Berechnung}

Auf der Eichkurve wird die der Probe-Extinktion entsprechende Menge Pregnandiol abgelesen. $\mathrm{Da} 1 \mathrm{ml}$ alkoholischer Extrakt benützt wurde, entspricht die auf der Eichkurve abgelesene Menge dem in $40 \mathrm{~m} l$ Harn enthaltenen Pregnandiol. Es wird auf die 24-Stdn.-Harnmenge umgerechnet.

\section{Ergebnisse}

Wiederauffindungsversuche mit Pregnandiol, das in Form von Na-Pregnandiol-Glucuronid $(6 \mathrm{mg} / \mathrm{l}) \mathrm{zu}$ $200-400 \mathrm{~m} l$ Harn zugesetzt wurde, ergaben eine Wiederauffindungsrate von $83,2-95,9 \%$.

Zwanzig Doppelbestimmungen bei einer PregnandiolKonzentration von $2,5 \mathrm{mg}$ pro $200 \mathrm{~m} /$ Harn ergaben eine durchschnittliche Abweichung der Einzelwerte von $4,4 \%$.
Die Empfindlichkeit der Methode liegt bei $0,2 \mathrm{mg} / 24$ Stdn.

Úber 100 mit der Methode von Sommervilue (15) parallel ausgeführten Bestimmungen ergaben Unterschiede von -3 bis $-12,8 \%$ gegenüber der genannten Technik. Diese Unterschiede müssen den nichtentfernten Harnchromogenen zugesschrieben werden, welche die Schwefelsäurereaktion negativ beeinflussen.

Sind kleine Mengen Pregnandiol vorhanden, so kann man statt $1 \mathrm{ml}$ alkoholischen Extrakt 2-5 $\mathrm{ml}$ dieses Extraktes einsetzen. Bei der Berechnung wird die Ausgangsmenge berücksichtigt.

Ein durch Luftabschluß durchgeführtes Abdestillieren des Toluols ergibt keine nennenswerte Unterschiede.

Der besondere Vorteil dieser Methode liegt in der Tatsache, daB man mit einer einfachen Reinigung des Extraktes (Alkaliwaschung) auskommt, denn die noch zurückgebliebenen Verunreinigungen scheiden, da sie selbst keine Farbreaktion mit dem Phospho-VanillinReagenz geben, aus. Ihre Einfachheit, Genauigkeit und gute Reproduzierbarkeit macht sie auch kleinen Laboratorien zugänglich. Wie bereits erwähnt, erbringt sie bei der UUberwachung labiler Schwangerschaften sehr gute Dienste, speziell zwischen der 6 . Woche und den 9. Monat der Schwangerschaft.

Die mit dieser Methode gefundenen Normalwerte für normal ablaufende Schwangerschaften sind in Tabelle 1 angegeben.

Tab. 1

Pregnandiolausscheidung in den verschiedenen Monaten normaler Schwangerschaften

\begin{tabular}{cc}
\hline $\begin{array}{c}\text { Dauer der } \\
\text { Schwangerschaft } \\
\text { Monate }\end{array}$ & $\begin{array}{c}\text { Pregnandiolausscheidung } \\
\text { (mg/24 Stdn.) }\end{array}$ \\
\hline $1^{1 / 2}$ & $8-13$ \\
2 & $10-15$ \\
3 & $14-18$ \\
4 & $16-24$ \\
5 & $24-30$ \\
6 & $28-41$ \\
7 & $38-48$ \\
8 & $45-57$ \\
9 & $50-60$ \\
\hline
\end{tabular}

\section{Literatur}

1. Klopper, A., A. E. Michie und J.B. Brown, J. Endocr. 12, 209 (1955). - 2. De Watteville, H., R. Barth und M. Gzell, J. clin. Endocr., Springfield 8, 962 (1948). - 3. GoLDzIEHER, W. und P. Nakamura, Acta endocr., K'hvn. 41, 371 (1962). - 4. Eberlein, W. R., A. M. Bongiovanni und C. M. Francts, J. Clin. Endocr., Springfield 18, 300 (1958). - 5. Martin, N. M., J. ReDDy und G. W. ThorN, J. Clin. Endocr., Springfield 21, 923 (1961). - 6. Starka, L. und J. Malikova, J. Endocr. 22, 215 (1961). - 7. Sommerville, I. F., Lancet, London 1948/II, 89. 8. Ilca, Sr., C. Dodica und Z. Ioanovicr, Z. analyt. Chem. 243, 289 (1968). - 9. MOLEN, H. J. VAN DER, Infrarood spectro- metrie van steroiden These, Utrecht, Neerlandia 1961. - 10. Henry, R. und M. Thévenet, Bull. Soc. Chim. Biol., Paris 41, 1391 (1959). - 11. Talbot, B. R., A. BermanN, E. A. MaC LACHLAN und J. K. WolfF, J. Clin. Endocr., Springfield 1, 668 (1941). - 12. Oertel, G. W. und E. Karser, Klin. Wschr. 31, 492 (1961). - 13. SAs, M., Klinische Anwendung der Sexualhormonbestimmungen, VEB Georg Thieme Verlag 158 (1963). 14. Zöllner N., und K. KIRSCH, Z. exper. Med. 135, 545 (1962). - 15. Sommervilite, I. F., G. F. Marrian und J. Kellar, Lancet 1948/I, 89.

Dipl. Ing. Stefan Ilca Lugoj, Str. Lenin 26 Rumänien 